\title{
Development of a method for recognizing biomedical entities in the texts of scientific articles
}

\author{
Stepan Derevyanchenko \\ Academy of Sciences \\ Novosibirsk, Russia \\ Novosibirsk State University \\ Novosibirsk, Russia sod97@yandex.ru \\ ORCID ID: 0000-0001-9433-8341
}

\begin{abstract}
In this work, the problem of recognizing named entities in the texts of scientific articles of biological orientation was solved. Using a combination of machine learning methods allowed us to achieve high recognition quality indicators.
\end{abstract}

\section{Keywords - neural network, prediction, machine learning}

\section{Introduction}

Currently, there are thousands of scientific journals in the world that publish research results in various fields of biology and medicine. Huge databases of patents are collected. The number of articles and patents increases exponentially over time. The analysis of such a huge number of experimental facts presented in text sources (scientific publications and patents) requires the use of automatic methods for extracting knowledge (text-mining). At the moment, most automatic methods of extracting knowledge are based on algorithms of computational linguistics and automatic text processing (Natural Language Processing). One of the most important tasks of modern computational linguistics is the task of recognizing named entities (Named Entity Recognition). In terms of its importance within this field, this task is comparable to the task of defining parts of speech in classical linguistics.

\section{Results}

In this paper, the problem of recognizing named entities is solved using the corpus of scientific articles from the PubMed Internet resource.

The paper considers a neural network approach for solving the problem of recognizing named entities. Specifically, a multi-layer recurrent neural network consisting of the following layers is used:

- Layer of trainable context-dependent vectors for words (Embeddings) [1]

- Bidirectional LSTM network [2]

- Fully connected layer (Linear)

In addition, classical machine learning models are used as a method for online verification of the neural network, which determines the presence of this entity in the text, which helps to significantly reduce the number of errors. the Russian

The paper compares the quality of logistic regression, naive Bayes, random Forest [4], and gradient boosting on trees (XGBoosting) [3]. As a result of the research, high quality metrics for recognizing biomedical entities (proteins, metabolites, organisms, diseases, etc.) were obtained.

\section{ACKNOWLEDGMENT}

The authors thank the Siberian Branch of the Russian Academy of Sciences for the budget project No. 032420190040-C-01.

\section{REFERENCES}

[1] T. Mikolov, I. Sutskever, K. Chen, G. S Corrado, J. Dean

[2] "Distributed Representations of Words and Phrases and their

[3] Compositionality" Advances in Neural Information Processing Systems, pp. 3111-3119, 2013

[4] S. Hochreiter, J. Schmidhuber "Long short-term memory" Neural computation, vol. 9 (8), pp. 1735-1780, 1997.

[5] Y. B. Yann LeCun Leon Bottou, P. Haffner "Gradient-based learning applied to document recognition" IEEE, 1998.

[6] L. Breiman "Random Forests" Machine Learning, vol. 45 (1), pp. 5-32, 2001 .

[7] K. S. Jones "A Statistical Interpretation of Term Specificity and its Application in Retrieval“" Journal of Documentation, vol. 60 (5), pp. 493-502, 2004 